\title{
FORUM
}

\section{A Case of Random Collision Probabilities}

\author{
E. M. Goodwin and J. F. Kemp
}

IN the contribution to the joint Royal Institute of Navigation/Royal Ocean Racing Club meeting on Safety of Navigation under Sail (p. 467), an expression was quoted for the random probability of collision in the case of a large, relatively fast ship passing through a concentration of smaller craft. It was not appropriate to discuss the derivation of the expression in the particular circumstances of the meeting but the methodology may be of some general interest and it is therefore outlined in this note.

It is required to derive an expression giving the number of collisions which a large, fast vessel can expect when transiting an area in which a number $(N)$ of yachts are navigating in random directions.

The yachts have length $l_{1}$, breadth $b_{1}$ and mean speed $v_{1}$. The large vessel has length $l_{2}$, breadth $b_{2}$ and speed $v_{2}$. All vessels are assumed to have rectangular waterplanes.

Figure I shows a yacht is on a course which intersects that of the larger ship at an angle $\theta . v_{1}$ and $v_{2}$ are the velocity vectors of the two vessels, and the ship is moving with relative velocity $V=v_{2}-v_{1}$ with respect to the yacht.

There will be a collision if there is an overlap between the projections of the two vessels onto a line perpendicular to the relative velocity vector. This is equivalent to saying that there will be a collision if the centre of the yacht falls within the area swept out by the sum of the two projections as the larger vessel advances along the relative velocity vector.

Taking angles $\alpha$ and $\beta$ as marked, the sum of the projections (P) for case I where $\beta$ is acute is given by:

$$
\begin{aligned}
P & =\left(l_{2} \sin \alpha+b_{2} \cos \alpha\right)+\left(I_{1} \sin \beta+b_{1} \cos \beta\right) \\
& =\frac{l_{2} v_{1} \sin \theta}{v}+b_{2} \sqrt{ }\left(1-\frac{v_{1} \sin ^{2} \theta}{v^{2}}\right)+\frac{l_{1} v_{2} \sin \theta}{v}+b_{1} \sqrt{\left(1-\frac{v_{2}^{2} \sin ^{2} \theta}{v^{2}}\right)}
\end{aligned}
$$

The rate $(R)$ at which area is swept out is the product of the above quantity and the relative velocity, $v$, i.e.

$$
\begin{aligned}
R= & I_{2} v \sin \theta+b_{2} \sqrt{ }\left(v^{2}-v_{1}^{2} \sin ^{2} \theta\right)+l_{1} v_{2} \sin \theta+b_{1} \sqrt{ }\left(v^{2}-v_{2} \sin ^{2} \theta\right) \\
= & I_{2} v_{1} \sin \theta+I_{1} v_{2} \sin \theta+b_{2} \sqrt{ }\left(v_{1}^{2}+v_{2}^{2}-2 v_{1} v_{2} \cos \theta-v_{1}^{2} \sin ^{2} \theta\right) \\
& +b_{1} \sqrt{ }\left(v_{1}{ }^{2}+v_{2}{ }^{2}-2 v_{1} v_{2} \cos \theta-v_{2}^{2} \sin ^{2} \theta\right) \\
= & I_{2} v_{1} \sin \theta+I_{1} v_{2} \sin \theta+b_{2} \sqrt{ }\left(v_{1}^{2} \cos ^{2} \theta+v_{2}^{2}-2 v_{1} v_{2} \cos \theta\right) \\
& +b_{1} \sqrt{ }\left(v_{2} \cos ^{2} \theta+v_{1}^{2}-2 v_{1} v_{2} \cos \theta\right) \\
= & I_{2} v_{1} \sin \theta+I_{1} v_{2} \sin \theta+b_{2}\left(v_{2}-v_{1} \cos \theta\right)+b_{1}\left(v_{1}-v_{2} \cos \theta\right)
\end{aligned}
$$

For case 2 , when $\beta$ is obtuse;

$$
P=\left(l_{2} \sin \alpha+b_{2} \cos \alpha\right)+\left(l_{1} \sin \beta-b_{1} \cos \beta\right)
$$



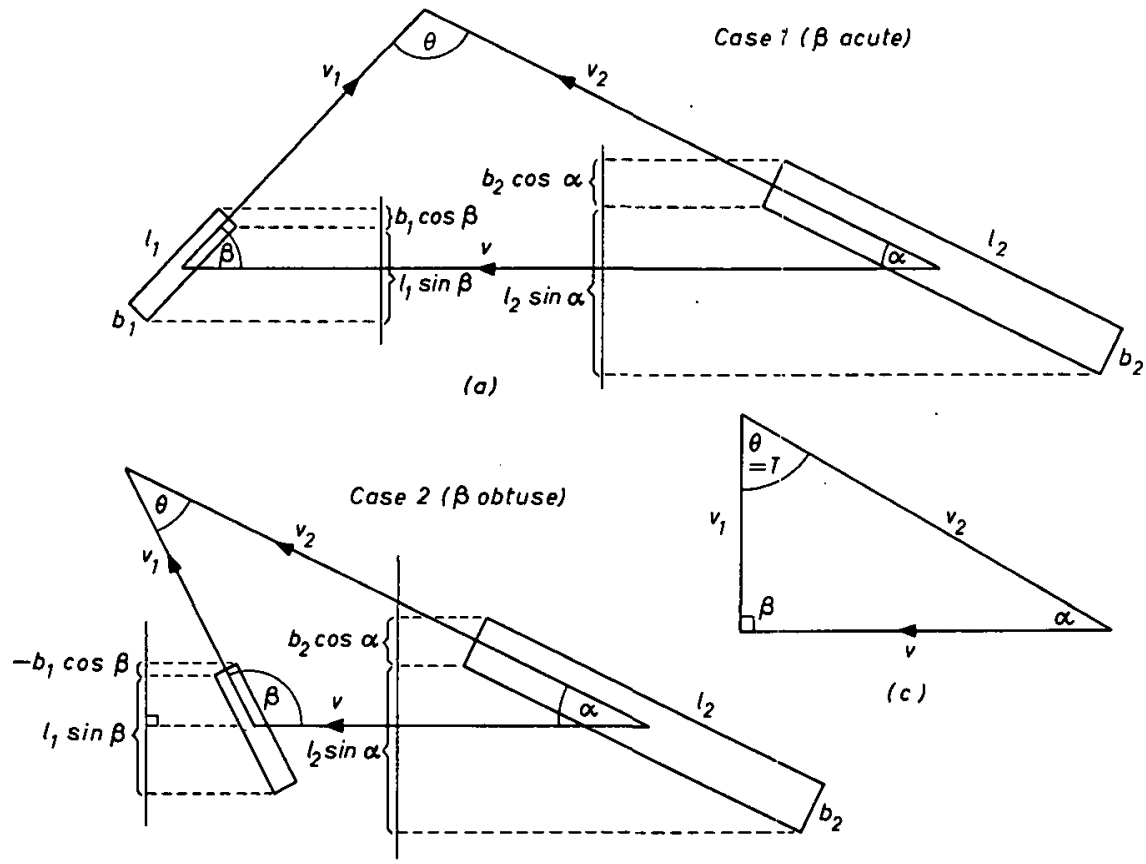

(b)

F1G. I .

and by a development similar to that for case $\mathbf{I}$ :

$$
R=l_{2} v_{1} \sin \theta+l_{1} v_{2} \sin \theta+b_{2}\left(v_{2}-v_{1} \cos \theta\right)-b_{1}\left(v_{1}-v_{2} \cos \theta\right)
$$

Consideration of Fig. I shows that the transition value of $\theta$, say $T$, between the case when $\beta$ is acute and the case when $\beta$ is obtuse occurs when

$$
\cos T=v_{1} / v_{2}
$$

The mean value of the rate $R_{\mathrm{m}}$ with respect to $\theta$, as $\theta$ varies from $\circ$ to $\pi$ is thus given by :

$$
\begin{aligned}
R_{\mathfrak{m}}= & \frac{I}{\pi}\left[\int_{0}^{T}\left\{I_{2} v_{1} \sin \theta+l_{1} v_{2} \sin \theta+b_{2}\left(v_{2}-v_{1} \cos \theta\right)-b_{1}\left(v_{1}-v_{2} \cos \theta\right)\right\} d \theta\right. \\
& \left.\quad+\int_{T}^{\pi}\left\{I_{2} v_{1} \sin \theta+I_{1} v_{2} \sin \theta+b_{2}\left(v_{2}-v_{1} \cos \theta\right)+b_{1}\left(v_{1}-v_{2} \cos \theta\right)\right\} d \theta\right] \\
= & \frac{1}{\pi}\left[2\left(I_{2} v_{1}+I_{1} v_{2}\right)+b_{2} v_{2} \pi+b_{1} v_{1} \pi+2 b_{1}\left(v_{2} \sin T-v_{1} T\right)\right]
\end{aligned}
$$

The mean number of expected collisions ( $n$ ) per unit time for the ship navigating across the area is the product of the rate at which the area is swept out and the mean density $(\rho)$ of the small vessels. Thus :

$$
n=R_{\mathrm{m}} \rho
$$

The total number of collisions $(M)$ to be expected for the ship passing through the area is the product of the collision rate and the transit time $(t)$. 
Thus:

$M=n t$

$$
\begin{aligned}
& =\frac{R \rho L}{v_{2}} \quad[\text { where } L \text { is the length of the large vessel's track through the area] } \\
& =\frac{\rho L}{\pi}\left[2\left(I_{1}+\lambda I_{2}\right)+b_{2} \pi+\lambda b_{1} \pi+2 b_{1}(\sin T-\lambda T)\right]
\end{aligned}
$$

[where $\lambda=\left(v_{1} / v_{2}\right)$ and $T=\cos ^{-1} \lambda$ ]

Note that when the yachts are small compared to the large vessel, the term $2 b_{1}$ $(\sin T-\lambda T)$ is very small and may be neglected. The expression then becomes:

$$
M=L \rho\left[\frac{2\left(I_{1}+\lambda I_{2}\right)}{\pi}+\left(b_{2}+\lambda b_{1}\right)\right]
$$

\section{Inertial Navigation}

\section{Claud Powell}

THE following letter to Nature, dated 24 April 1873 , might make an appropriate sequel to 'The history of inertial navigation' by W. Wrigley in the January issue of the Journal. As well as containing some interesting speculation about animal navigation, which we seem no nearer resolving a century later, the letter includes an introduction to the idea of inertial navigation that it would be difficult to improve upon even today.

\section{Instinct : A Mechanical Analogy}

Mr. Darwin, in his article on 'The Origin of certain Instincts,' in Nature of April 3 , appears inclined to think that what we may call the instinct of direction in animals is of the same kind as the faculty by which men find their way: and he instances the power of the natives of Siberia to find their way over hummocky ice. He afterwards, however, raises without discussing the question 'whether animals may not possess the faculty of keeping a dead reckoning of their course in a much more perfect degree than man, or whether this faculty may not come into play on the commencement of a journey when an animal is shut up in a basket.' I wish to point out that this peculiar power of animals is one that cannot be explained as a higher degree of any power that man possesses. What man can do is to find the third side of a triangle after travelling the other two sides with his eyes open. Animals can do the same after travelling the two sides with their eyes shut. The former power of man here spoken of depends on the careful use of his powers of observation. This does not appear to be the case with animals. Among the many instances of animals finding their way home after being conveyed away without any opportunity of seeing their way or taking their bearings, there must in all probability be many in which the animal slept on the journey: and if so, the mental or organic process whereby it was able to know its way back must have gone on during sleep. There is nothing in man's 